\title{
Optical spectroscopy and electronic structure of the face-centered icosahedral quasicrystals $\mathrm{Zn}$ - $\operatorname{Mg}-\boldsymbol{R}(\boldsymbol{R}=\mathrm{Y}, \mathrm{Ho}, \mathrm{Er})$
}

\author{
V Karpus, S Tumenas, A Suchodolskis, Hans Arwin and W Assmus
}

\section{Linköping University Post Print}

\section{Tweet}

N.B.: When citing this work, cite the original article.

Original Publication:

V Karpus, S Tumenas, A Suchodolskis, Hans Arwin and W Assmus, Optical spectroscopy and electronic structure of the face-centered icosahedral quasicrystals $\mathrm{Zn}-\mathrm{Mg}-R(R=\mathrm{Y}$, Ho, Er), 2013, Physical Review B. Condensed Matter and Materials Physics, (88), 9. http://dx.doi.org/10.1103/PhysRevB.88.094201

Copyright: American Physical Society http://www.aps.org/ 


\title{
Optical spectroscopy and electronic structure of the face-centered icosahedral quasicrystals Zn-Mg- $R(R=$ Y, Ho, Er $)$
}

\author{
V. Karpus, ${ }^{1, *}$ S. Tumenas, ${ }^{1}$ A. Suchodolskis, ${ }^{1}$ H. Arwin, ${ }^{2}$ and W. Assmus ${ }^{3}$ \\ ${ }^{1}$ Center for Physical Sciences and Technology, Semiconductor Physics Institute, A. Goštauto 11, LT-01108 Vilnius, Lithuania \\ ${ }^{2}$ Laboratory of Applied Optics, Department of Physics, Chemistry and Biology, Linköping University, SE-58183 Linköping, Sweden \\ ${ }^{3} J$. W. Goethe-Universität, Physikalisches Institut, Max-von-Laue-Str. 1, D-60438 Frankfurt am Main, Germany
}

(Received 2 July 2013; revised manuscript received 27 August 2013; published 9 September 2013)

\begin{abstract}
Results of optical spectroscopy studies of the face-centered icosahedral (fci) single-grain Zn-Mg-Y, Zn-Mg-Ho, and $\mathrm{Zn}-\mathrm{Mg}$-Er quasicrystals (QCs) are presented. The dielectric function of the QCs was measured in the 0.01-6 eV spectral range by IR-UV spectroscopic ellipsometry and far infrared reflection spectroscopy techniques. A theoretical scheme of optical conductivity calculations is extended to account for the Fermi level positions within and below a pseudogap. The model of the QC electron energy spectrum, based on a band structure hypothesis, is suggested, which treats the electronic subsystem as a nearly free electron gas affected by intersections of the Fermi surface with several families of Bragg planes. The experimental optical spectra are reproduced in detail by theoretical calculations carried out within the framework of the model. The parameters of the electron energy spectrum deduced from an analysis of optical data are close to those previously determined in an analysis of fci $\mathrm{Zn}-\mathrm{Mg}-R$ valence band photoemission spectra.
\end{abstract}

DOI: 10.1103/PhysRevB.88.094201

PACS number(s): 71.23.Ft

\section{INTRODUCTION}

Investigations of the optical response of quasicrystals (QCs) is one of the principal tools for determination of their electronic structure. Optical studies of icosahedral Al-Mn, ${ }^{1}$ $\mathrm{Al}-\mathrm{Cu}-\mathrm{Fe},{ }^{2} \mathrm{Al}-\mathrm{Cu}-\mathrm{Fe}-\mathrm{B},{ }^{3} \mathrm{Al}-\mathrm{Pd}-\mathrm{Mn},{ }^{3-5} \mathrm{Al}-\mathrm{Pd}-\mathrm{Re},{ }^{6-8}$ and $\mathrm{Al}-$ $\mathrm{Mn}-\mathrm{Si}^{9}$ quasicrystals reveal the main features of their optical spectra to be the broad absorption peak at about $1-2 \mathrm{eV}$ and a strong suppression of the usual metallic Drude-type optical transitions. Investigations of decagonal quasicrystalline Al$\mathrm{Co}-\mathrm{Cu},{ }^{10} \mathrm{Al}-\mathrm{Co}-\mathrm{Cu}-\mathrm{Si},{ }^{10}$ and $\mathrm{Al}-\mathrm{Ni}-\mathrm{Co}^{11}$ phases show an essential reduction of the Drude-type response in the plane of quasicrystalline atomic arrangement as compared to the optical response in the periodic, crystalline direction.

Our previous preliminary spectroscopic ellipsometry studies $^{12-14}$ of icosahedral $\mathrm{Zn}-\mathrm{Mg}-R$ quasicrystals (where $R=\mathrm{Y}$, Ho, Er), as well as reflection spectroscopy studies of $\mathrm{Zn}-\mathrm{Mg}-\mathrm{Y}$ and $\mathrm{Zn}-\mathrm{Mg}-\mathrm{Tb}$ by Chernikov et al. ${ }^{15}$ revealed a strong interband optical feature at about $1 \mathrm{eV}$, but a larger Drude peak as compared to that in Al-based quasicrystals.

A distinctive feature of the $\mathrm{Zn}-\mathrm{Mg}-R$ quasicrystals with respect to most other QCs is the fact that their valence bands are due predominantly to the $s p$-type electron states, and from this point of view they are closer to simple metals. This can be one of the reasons for the comparatively higher Drude contribution in $\mathrm{Zn}-\mathrm{Mg}-R$.

The $s p$ character of the $\mathrm{Zn}-\mathrm{Mg}-R$ valence bands was revealed by their photoemission (PE) studies. ${ }^{16-18}$ On the basis of the PE data analysis, a model for the $\mathrm{Zn}-\mathrm{Mg}-R$ electron energy spectrum was suggested, ${ }^{16}$ which allowed for a successful description of the experimental $\mathrm{Zn}-\mathrm{Mg}-R$ valence band PE spectra. The model is based on a band structure hypothesis and treats the $\mathrm{Zn}-\mathrm{Mg}-R$ electronic subsystem as a nearly free-electron (NFE) gas, affected by a weak quasicrystalline potential. The hypothetical $\mathrm{Zn}-\mathrm{Mg}-R$ electron energy spectrum in the vicinity of the Fermi level is determined by the Fermi surface intersections with several families of Bragg planes.
The suggested model for the electronic structure of $\mathrm{Zn}-\mathrm{Mg}$ $R$ was applied to the analysis of optical spectra of the simple icosahedral Zn-Mg-Ho quasicrystal ${ }^{19}$ and the face-centered icosahedral (fci) Zn-Mg- $R\left(R=\mathrm{Y}\right.$, Ho, Er) QCs. ${ }^{20}$ The theoretical optical spectra, simulated within the framework of the model, qualitatively agreed with the experimental spectra.

Here we will present (Sec. II) new experimental data for the optical response of $\mathrm{Zn}-\mathrm{Mg}-R$ recorded by IR-UV spectroscopic ellipsometry and FIR reflection spectroscopy techniques, which allowed us to expand the spectral range of $\mathrm{Zn}-\mathrm{Mg}-R$ dielectric function and optical conductivity from the previous window ${ }^{19,20}$ of $0.1-6 \mathrm{eV}$ to the $0.01-6 \mathrm{eV}$ range. Additionally, we have employed a surface-preparation plasma-etching technique, which leads to an increase in the optical features in the new IR-UV data compared to those recorded previously.

The NFE-model of the $\mathrm{Zn}-\mathrm{Mg}-R$ electron energy spectrum will be further developed (Sec. III). The algorithm of the optical conductivity calculations, worked out by Ashcroft and Sturm, ${ }^{21}$ will be extended (Sec. IV) to account for various positions of the Fermi level with respect to a pseudogap, appropriate for the $\mathrm{Zn}-\mathrm{Mg}-R$ quasicrystals under consideration.

Finally (Sec. V), the suggested electron energy spectrum model will be applied for a detailed analysis of the experimental Zn-Mg- $R$ optical conductivity spectra. The spectra can be nicely explained within the framework of the theoretical model. The parameters of the electron energy spectrum, deduced from the optical data analysis, are in close agreement with those obtained in an analysis of $\mathrm{Zn}-\mathrm{Mg}-R$ photoemission spectroscopy data.

\section{EXPERIMENT}

The fci single-grain $\mathrm{Zn}_{62} \mathrm{Mg}_{29} \mathrm{Y}_{9}, \mathrm{Zn}_{65} \mathrm{Mg}_{25} \mathrm{Ho}_{10}$, and $\mathrm{Zn}_{65} \mathrm{Mg}_{24} \mathrm{Er}_{11}$ quasicrystals were grown by the liquidencapsulated top-seeded solution-growth method. ${ }^{22,23}$ The $\mathrm{x}$-ray diffraction patterns of the QCs consist of sharp, instrumental resolution limited Bragg peaks (Fig. 1) indicating a 


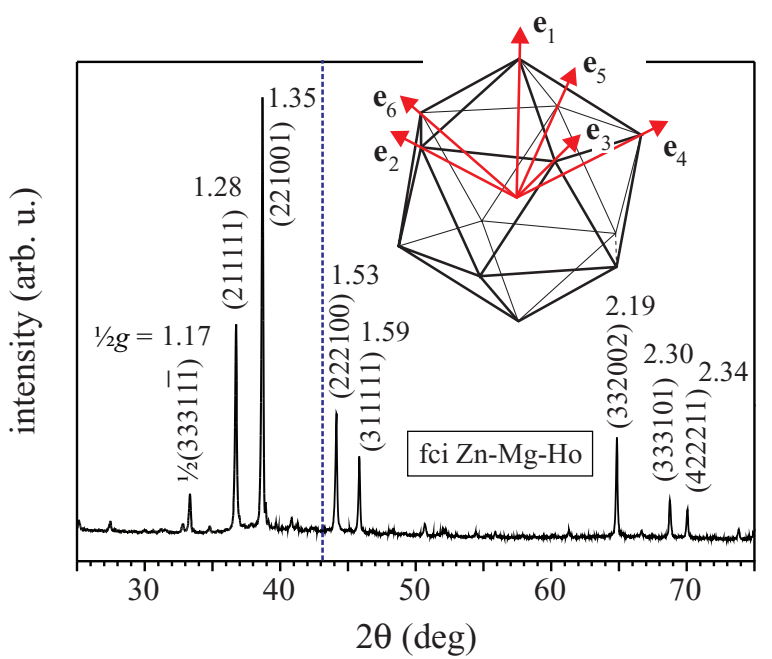

FIG. 1. (Color online) X-ray powder diffractogram of a Zn-MgHo quasicrystal. Indices of the diffraction peaks correspond to indices of the reciprocal lattice vectors $\boldsymbol{g}=\frac{\pi}{a} \sum_{i} m_{i} \mathbf{e}_{i}$. Numbers at the peaks indicate half moduli of the $g$ vectors in $10^{8} \mathrm{~cm}^{-1}$ units. Dashed line corresponds to the Fermi wave vector (Table I).

high structural quality of the quasicrystals. The mass density $\varrho$ of the QCs was measured by the Micrometrics AccuPyc 1330 setup. The valence electron concentrations, determined from the recorded $\varrho$ values, are presented in Table I.

The optical surfaces of single-grain QC samples were prepared by a careful mechanical polishing as described in our previous work. ${ }^{20}$ Additionally, as a final surface-preparation procedure, we employed a plasma etching technique by $\mathrm{Ar}^{+}$ ion sputtering in UHV chamber. Figure 2 illustrates the effect of the final plasma etching on the $\mathrm{Zn}-\mathrm{Mg}$-Ho optical conductivity spectrum. The etching allowed for an increase of $\mathrm{Zn}-\mathrm{Mg}-R$ optical features by about $10 \%$.

The IR-UV dielectric function $\varepsilon(\omega)$ of the quasicrystals was measured with spectroscopic ellipsometry (SE). In the spectral range $0.73-6 \mathrm{eV}$, the measurements were carried out with a dual rotating compensators ellipsometer (RC2, J. A. Woollam Co., Inc.) in steps of $1 \mathrm{~nm}$ at 50, 60, and 70 degrees of the incident angle. In the IR spectral region $333-5000 \mathrm{~cm}^{-1}$, a rotating-compensator Fourier-transform ellipsometer (IRSE, J. A. Woollam Co., Inc.) with a resolution of $4 \mathrm{~cm}^{-1}$ was used at the same angles of incidence. The measurements allowed for a reliable determination of $\varepsilon(\omega)$ on a wavelength-by-wavelength basis in the spectral range from ca. $0.15 \mathrm{eV}$ up to $6 \mathrm{eV}$ (dots in Fig. 3).

TABLE I. Physical parameters of fci Zn-Mg- $R$ quasicrystals: the mass density $\varrho$, the valence electron concentration $n$, and the Fermi wave vector $k_{\mathrm{F}}=\left(3 \pi^{2} n\right)^{1 / 3}$.

\begin{tabular}{lccc}
\hline \hline & $\begin{array}{c}\varrho \\
\left(\mathrm{g} / \mathrm{cm}^{3}\right)\end{array}$ & $\begin{array}{c}n \\
\left(10^{23} \mathrm{~cm}^{-3}\right)\end{array}$ & $\begin{array}{c}k_{\mathrm{F}} \\
\left(10^{-8} \mathrm{~cm}^{-1}\right)\end{array}$ \\
\hline $\mathrm{Zn}_{62} \mathrm{Mg}_{29} \mathrm{Y}_{9}$ & 5.18 & 1.17 & 1.51 \\
$\mathrm{Zn}_{65} \mathrm{Mg}_{25} \mathrm{Ho}_{10}$ & 5.82 & 1.13 & 1.50 \\
$\mathrm{Zn}_{65} \mathrm{Mg}_{24} \mathrm{Er}_{11}$ & 5.87 & 1.12 & 1.49 \\
\hline \hline
\end{tabular}

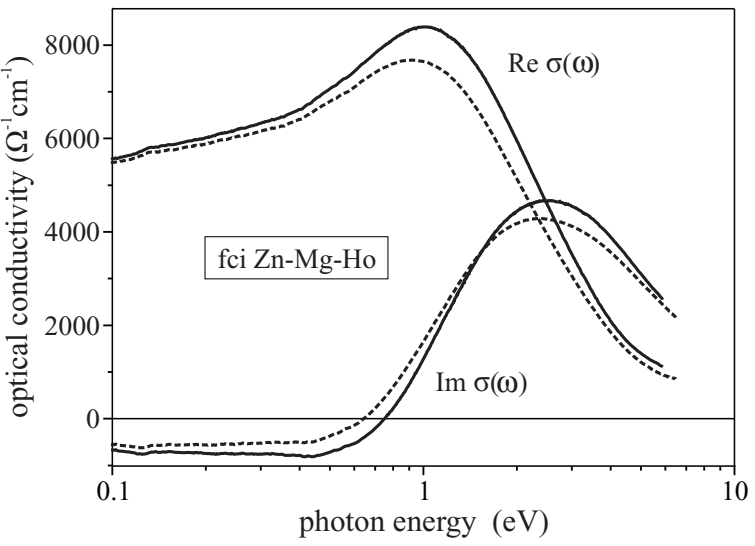

FIG. 2. Influence of the $\mathrm{Ar}^{+}$etching on the $\mathrm{Zn}-\mathrm{Mg}$-Ho optical conductivity spectrum. Dashed and solid curves present data recorded before and after sputtering, respectively.

In the spectral range $0.01-0.2 \mathrm{eV}$, the far infrared (FIR) optical response of $\mathrm{Zn}-\mathrm{Mg}-R$ quasicrystals was measured by Fourier-transform (FT) reflectance spectroscopy. The measurements were carried out with a Vertex $70 \mathrm{v}$ vacuum FT spectrometer with spectral resolution $4 \mathrm{~cm}^{-1}$. The FIR reflectivity spectrum $R(\omega)$ of $\mathrm{Zn}-\mathrm{Mg}-\mathrm{Y}$, appended in the IR-UV region by SE data, is presented by dots in Fig. 4. The full curve presents for a comparison data from Chernikov et al. ${ }^{15}$ As seen, the present $R(\omega)$ spectrum is close to that of Chernikov et al. in the long-wavelength region. However, it shows higher reflectance in the VIS-UV range, which we suppose is due to either different optical surface preparation techniques used or different atomic compositions of investigated $\mathrm{Zn}-\mathrm{Mg}-\mathrm{Y}$ samples.

The $\mathrm{Zn}-\mathrm{Mg}-R$ dielectric function $\varepsilon(\omega)$ in FIR region (presented by curves in Fig. 3) was determined from the $R(\omega)$ spectrum with a Kramers-Kronig transform making use of the anchor-window technique. ${ }^{24}$ The high-frequency $R(\omega)$ asymptote (dashed curve in Fig. 4) was determined from the requirement that the dielectric function determined by the Kramers-Kronig transform would coincide with SE data in IR-UV range.

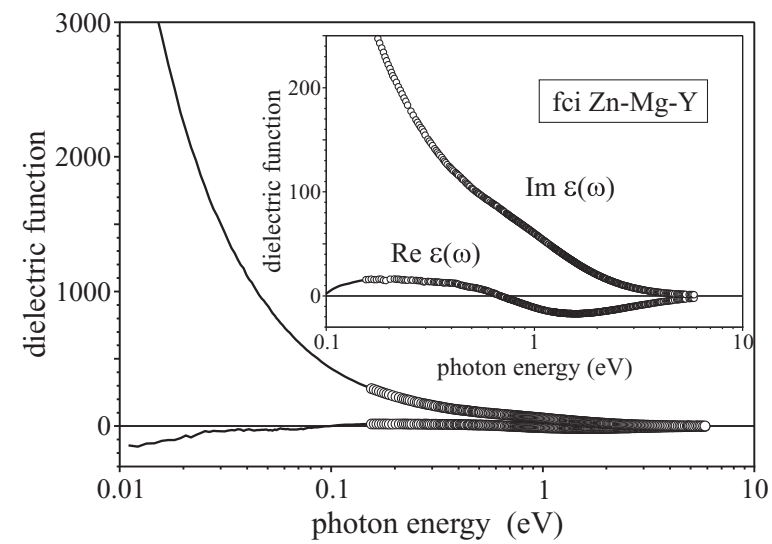

FIG. 3. Dielectric function of $\mathrm{Zn}-\mathrm{Mg}-\mathrm{Y}$ quasicrystal. (Dots present spectroscopic ellipsometry data, curves correspond to $\varepsilon(\omega)$ determined by the Kramers-Kronig analysis of FIR reflectivity spectrum.) 


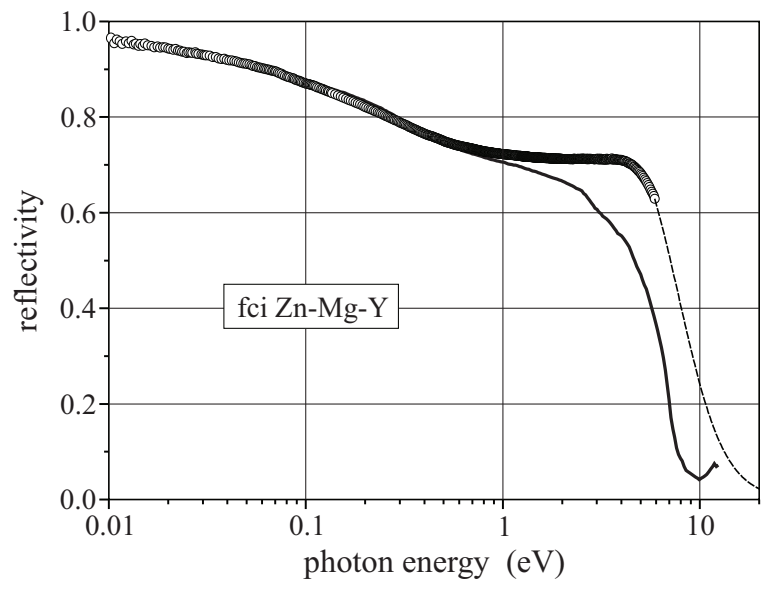

FIG. 4. Reflectivity spectrum of the $\mathrm{Zn}-\mathrm{Mg}-\mathrm{Y}$ quasicrystal. (Dots present our experimental data, full curve presents literature data, ${ }^{15}$ dashed curve corresponds to the high-frequency extrapolation used in Kramers-Kronig analysis.)

\section{MODEL OF ELECTRON ENERGY SPECTRUM}

Since the Bloch theorem is not applicable for aperiodic systems, the problem of an electronic structure of quasicrystals, despite of its importance, remains to be in principle unsolved. The QC electronic structure is usually considered in frameworks of two different approaches.

The first one treats the quasicrystalline potential acting on the electron subsystem as that of the QC approximants. This reduces the QC electronic structure problem to that of periodic crystals with huge unit cells, containing large numbers of atoms, and relevant techniques of band-structure calculations, usually the tight-binding linear-muffin-tin-orbital (TB-LMTO) method, are being employed to examine the QC valence bands (see, e. g., reviews 25-27). The calculations predict a spiky structure of the valence band density of states (DOS) and a pseudogap feature at the Fermi level.

The alternative approach, the so-called band structure hypothesis, ${ }^{28}$ treats the electronic subsystem in quasicrystals as a NFE-gas, affected by a weak quasicrystalline potential

$$
V(\boldsymbol{r})=\sum_{\boldsymbol{g}} V_{\boldsymbol{g}} \exp (i \boldsymbol{g} \cdot \boldsymbol{r}) .
$$

(Here $\boldsymbol{g}$ are the reciprocal lattice vectors.) The approach, when accounting for several Fourier amplitudes $V_{g}$ of the QC potential only, yields as well a pseudogap structure, but otherwise a smooth DOS dispersion.

Though the DOS spikiness is considered to be a distinctive feature of quasicrystals, it was not observed experimentally. This can be due to a finite broadening/scattering parameter $\Gamma=$ $\hbar / \tau$ of electron states. As has been shown by Fujiwara et al. ${ }^{29}$ a value of $\Gamma=0.7 \mathrm{eV}$ completely washes out an anticipated spiky structure of the optical conductivity spectrum. Since the relaxation times in all known quasicrystals are short and correspond to $\Gamma$ of the order of $0.1-1 \mathrm{eV}$, experimentally one can not unambiguously distinguish between the two electron energy spectrum models.

In our previous $\mathrm{Zn}-\mathrm{Mg}-\mathrm{R}$ PE studies, ${ }^{16-18}$ the bandstructure hypothesis approach was successfully applied for an analysis of the valence-band PE spectra. The $\mathrm{Zn}-\mathrm{Mg}-R$
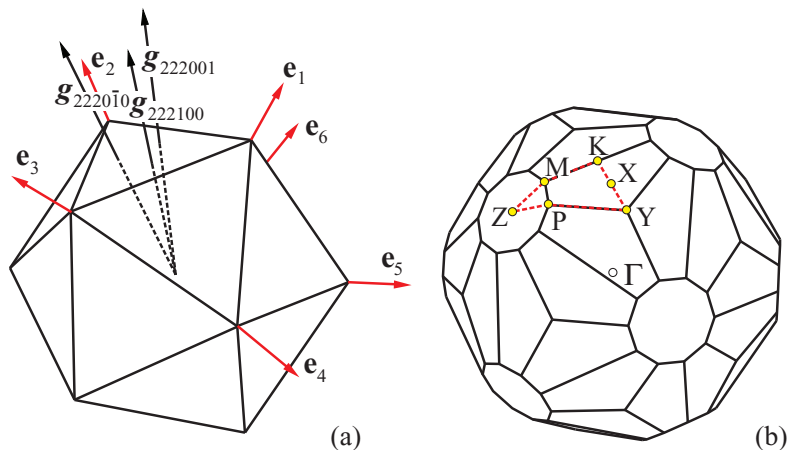

FIG. 5. (Color online) Orientation of $\boldsymbol{g}_{222100}, \boldsymbol{g}_{222001}$, and $\boldsymbol{g}_{2220 \overline{1} 0}$ vectors (a) and the effective 222100 and 311111 Brillouin zone (b).

valence-band density of states was calculated in the framework of the NFE approximation and the calculated DOS nicely reproduced the observed PE valence-band spectra. Moreover, the calculations ${ }^{16}$ yielded a correct experimental value of the Zn-Mg-Y electronic specific-heat coefficient and agreed well with the main $\mathrm{Zn}-\mathrm{Mg}-\mathrm{Y}$ DOS features, independently calculated by the TB-LMTO technique. ${ }^{30,31}$

\section{A. NFE approximation}

The model of the $\mathrm{Zn}-\mathrm{Mg}-R$ electronic structure, which was used for an analysis of the PE data, is based on the NFE approximation and treats the quasicrystalline potential (1) as a small perturbation.

Though the reciprocal quasicrystalline lattice is dense, the structure factors $S_{g}$ of most of its nodes are very low. The $\boldsymbol{g}$ vectors, which play a decisive role in the quasicrystalline potential (1), correspond to both high structure factors and the Bragg planes, which are in the proximity of the Fermi surface. This set of $\boldsymbol{g}$ vectors, which we will denote as $\mathcal{G}$, can be determined from the experimental diffraction patterns.

In $\mathrm{Zn}-\mathrm{Mg}-R$ quasicrystals, $\mathcal{G}$ is comprised (see Fig. 1) of twelve $\boldsymbol{g}_{311111}$ vectors, which are directed along the $C_{5}$ axes of icosahedron, and sixty $\boldsymbol{g}_{222100}$ vectors. The subset of $\boldsymbol{g}_{222100}$ vectors is constituted of twenty triads, which group around $C_{3}$ axes, as illustrated in Fig. 5(a) for the $\left\{\boldsymbol{g}_{222100}, \boldsymbol{g}_{222001}, \boldsymbol{g}_{2220 \overline{10}}\right\}$ triad around $\boldsymbol{g}_{222000} \| C_{3}$. The $72 \boldsymbol{g} \in \mathcal{G}$ vectors determine the effective Brillouin zone of $\mathrm{Zn}-\mathrm{Mg}-R$ quasicrystals, ${ }^{16,17}$ the faces of which correspond to the $g \in \mathcal{G}$ Bragg planes [Fig. 5(b)].

The electron energy spectrum problem can be simplified by leaving only the $\boldsymbol{g} \in \mathcal{G}$ terms in the Fourier expansion of the quasicrystalline potential (1),

$$
V(\boldsymbol{r})=\sum_{\boldsymbol{g} \in \mathcal{G}} V_{\boldsymbol{g}} \exp (i \boldsymbol{g} \cdot \boldsymbol{r}),
$$

and in the expansion of the electron wave function

$$
\Psi_{k}=C_{k} \Phi_{k}+\sum_{g \in \mathcal{G}} C_{k-g} \Phi_{k-g} .
$$

Here $\Phi_{\boldsymbol{k}}=\Omega^{-1 / 2} \exp (i \boldsymbol{k} \cdot \boldsymbol{r})$ are the plane waves, and $\Omega$ is the normalization volume. The Fourier amplitude $V_{0}$ of the quasicrystalline potential (1) was eliminated by a shift of the energy reference point. 
The standard procedure of a projection of the Schrödinger equation onto the $\Phi_{k}$ and $\Phi_{k-g}$ eigenfunctions yields the following system of equations for coefficients of the wavefunction expansion (3):

$$
\begin{gathered}
{\left[\epsilon_{\boldsymbol{k}}-\varepsilon(\boldsymbol{k})\right] C_{\boldsymbol{k}}+\sum_{\boldsymbol{g} \in \mathcal{G}} V_{g} C_{k-g}=0,} \\
{\left[\epsilon_{\boldsymbol{k}-\boldsymbol{g}}-\varepsilon(\boldsymbol{k})\right] C_{\boldsymbol{k}-\boldsymbol{g}}+V_{-g} C_{\boldsymbol{k}}=0 .}
\end{gathered}
$$

Here $\varepsilon(\boldsymbol{k})$ is the electron energy to be determined and $\epsilon_{\boldsymbol{k}}=\hbar^{2} k^{2} / 2 m_{0}$ is the free electron energy. In a derivation of Eqs. (4) and (5), it was taken into account that a difference of any two $\boldsymbol{g}$ vectors of $\mathcal{G}, \boldsymbol{g}_{1} \in \mathcal{G}$ and $\boldsymbol{g}_{2} \in \mathcal{G}$, does not belong to $\mathcal{G}, \boldsymbol{g}_{1}-\boldsymbol{g}_{2} \notin \mathcal{G}$.

Equation (5) can be easily solved with respect to $C_{\boldsymbol{k}-\boldsymbol{g}}$, and by inserting the answer into (4) one obtains the dispersion equation

$$
\varepsilon(\boldsymbol{k})-\epsilon_{\boldsymbol{k}}=\sum_{\boldsymbol{g} \in \mathcal{G}} \frac{\left|V_{\boldsymbol{g}}\right|^{2}}{\left(\varepsilon(\boldsymbol{k})-\epsilon_{\boldsymbol{k}}\right)-\left(\epsilon_{\boldsymbol{k}-\boldsymbol{g}}-\epsilon_{\boldsymbol{k}}\right)},
$$

which determines the electron energy spectrum.

The dispersion equation (6) can be solved numerically at given values of two parameters, the $V_{222100}$ and $V_{311111}$ Fourier amplitudes of the quasicrystalline potential (2). The hypothetical $\mathrm{Zn}-\mathrm{Mg}-R$ energy spectrum, calculated at the $V_{222100}=0.31 \mathrm{eV}$ and $V_{311111}=0.58 \mathrm{eV}$ values determined in an analysis of the experimental $\mathrm{Zn}-\mathrm{Mg}$-Y PE valence-band spectra, ${ }^{16}$ is presented in Fig. 6. Since the $V_{222100}$ and $V_{311111}$ pseudopotentials acquire similar values in $\mathrm{Zn}-\mathrm{Mg}-\mathrm{Y}, \mathrm{Zn}-\mathrm{Mg}-$ Ho, and $\mathrm{Zn}-\mathrm{Mg}$-Er quasicrystals, ${ }^{16,17}$ the energy spectrum (Fig. 6) can be considered as a hypothetical band structure in the vicinity of the Fermi level for all three, Zn-Mg-Y, $\mathrm{Zn}-\mathrm{Mg}-\mathrm{Ho}$, and $\mathrm{Zn}-\mathrm{Mg}-\mathrm{Er}$, quasicrystals studied. The energy spectrum in Fig. 6 is presented in $\varepsilon_{0}=\hbar^{2}\left(g_{222100} / 2\right)^{2} / 2 m_{0}$ units and is depicted along the KXYPZMK path on faces of the effective Brillouin zone, indicated in Fig. 5(b).

As seen in Fig. 6, the Fermi level is above the $\Delta_{222100}=$ $2\left|V_{222100}\right|$ pseudogap and lies in a lower part of the $\Delta_{311111}=$ $2\left|V_{311111}\right|$ pseudogap. The lower energy band is almost

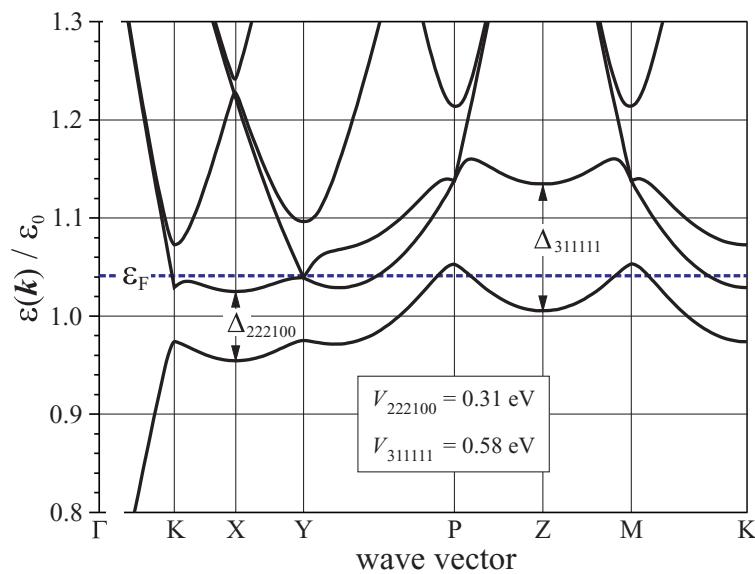

FIG. 6. (Color online) Energy spectrum of $\mathrm{Zn}-\mathrm{Mg}-R$ quasicrystals in the vicinity of the Fermi level, at the pseudopotential values $V_{222100}=0.31 \mathrm{eV}, V_{311111}=0.58 \mathrm{eV}$, and the Fermi energy $\varepsilon_{\mathrm{F}}=$ $9.31 \mathrm{eV}$ determined from an analysis of the experimental $\mathrm{Zn}-\mathrm{Mg}-\mathrm{Y}$ PE data. ${ }^{16}$ completely filled with electrons, the empty states, the hole pockets, are left along lines connecting $\mathrm{P}$ and $\mathrm{M}$ points only. The electrons fill up the higher energy band along the KY lines. These features of the energy spectrum suggest a presumable semimetallic behavior of the $\mathrm{Zn}-\mathrm{Mg}-\mathrm{R}$ quasicrystals. This is in agreement with results of Hall effect measurements on $\mathrm{Zn}-\mathrm{Mg}$ $\mathrm{Y},{ }^{15}$ which indicate that the effective electron concentration is very low, $n_{\text {eff }}=2.3 \times 10^{21} \mathrm{~cm}^{-3}$, as compared to the valence electron concentration $n=1.17 \times 10^{23} \mathrm{~cm}^{-3}$ (Table I).

The $\mathrm{Zn}-\mathrm{Mg}-\mathrm{R}$ energy spectrum scheme presented in Fig. 6 allows for a prediction of the interband optical transitions across $\Delta_{222100}$ and $\Delta_{311111}$ pseudogaps, which should result in the optical feature at about $1 \mathrm{eV}$. The optical spectra, in principle, can be numerically calculated making use of the presented hypothetical band-structure scheme. However, since only particular regions of $\boldsymbol{k}$ space (where the electron energy spectrum deviates from the free-electron one) are of importance for optical transitions, the analytical calculations, which take into account a detailed description of the energy spectrum in the vicinity of pseudogaps, are much more effective.

\section{B. Model of independent intersections}

The energy spectrum of the NFE gas is essentially affected by the quasicrystalline potential at intersections of the isoenergetic surface with Bragg planes. In proximity to a chosen Bragg plane, the dispersion equation (6) can be simplified-only a single term, corresponding to the plane, is sufficient to retain on its right-hand side,

$$
\delta \varepsilon_{\boldsymbol{g}}(\boldsymbol{k})=\frac{\left|V_{\boldsymbol{g}}\right|^{2}}{\delta \varepsilon_{\boldsymbol{g}}(\boldsymbol{k})-\left(\epsilon_{\boldsymbol{k}-\boldsymbol{g}}-\epsilon_{\boldsymbol{k}}\right)} .
$$

Here $\delta \varepsilon_{\boldsymbol{g}}(\boldsymbol{k})=\varepsilon(\boldsymbol{k})-\epsilon_{\boldsymbol{k}}$ is the deviation from the free electron spectrum due to an intersection with a single Bragg plane.

Solutions of Eq. (7) correspond to two well-known NFE energy spectrum branches (see, e. g., Ref. 32), which in the extended zone presentation acquire the form

$$
\delta \varepsilon_{\boldsymbol{g}}(\boldsymbol{k})=\frac{\Delta_{g}}{2}\left(\gamma-\frac{\gamma}{|\gamma|} \sqrt{\gamma^{2}+1}\right) .
$$

Here $\Delta_{g}=2\left|V_{g}\right|$ is the pseudogap, $\gamma$ is a function of the quasimomentum $\boldsymbol{k}$ component $k_{\|}$along the $\boldsymbol{g}$ direction,

$$
\gamma=\frac{\epsilon_{k-g}-\epsilon_{k}}{\Delta_{g}}=\frac{4 \varepsilon_{0}}{\Delta_{g}}\left(1-\frac{k_{\|}}{\frac{1}{2} g}\right),
$$

and $\varepsilon_{0}=\hbar^{2}\left(\frac{1}{2} g\right)^{2} / 2 m_{0}$ is the intersection energy.

In the extended zone presentation, used in the present study, the deviations $\delta \varepsilon_{g}(\boldsymbol{k})$ are localized in close proximity to Bragg planes, within the narrow strips the width of which, when $k_{\|}$is measured in $\frac{1}{2} g$ units, is about $\Delta_{g} / 4 \varepsilon_{0}$. Under the assumption that strips do not overlap, the electron energy spectrum, determined by Eq. (6), can be presented in the simple form

$$
\varepsilon(\boldsymbol{k})=\epsilon_{\boldsymbol{k}}+\sum_{\boldsymbol{g} \in \mathcal{G}} \delta \varepsilon_{\boldsymbol{g}}(\boldsymbol{k}) .
$$

Within the model of independent intersections, the coefficients $C_{\boldsymbol{k}-\boldsymbol{g}}$ of the wave-function expansion (3) are found from 
(5) by replacing $\varepsilon(\boldsymbol{k}) \rightarrow \epsilon_{\boldsymbol{k}}+\delta \varepsilon_{\boldsymbol{g}}(\boldsymbol{k})$,

$$
C_{k-g}=\frac{V_{-g}}{\mid V_{g \mid}}\left(\gamma-\frac{\gamma}{|\gamma|} \sqrt{\gamma^{2}+1}\right) C_{k},
$$

whereas the $C_{\boldsymbol{k}}$ coefficient is found from the normalization condition,

$$
\left|C_{\boldsymbol{k}}\right|^{2}=\left[1+\sum_{\boldsymbol{g} \in \mathcal{G}}\left(\gamma-\frac{\gamma}{|\gamma|} \sqrt{\gamma^{2}+1}\right)^{2}\right]^{-1} .
$$

The model of independent intersections breaks down at edges and vertices of the effective Brillouin zone. Since they have considerably smaller phase space than the BZ faces, the model should be a relevant tool for a description of optical transitions. However, one should keep in mind that an overlap of the strips, within which the deviations $\delta \varepsilon_{\boldsymbol{g}}(\boldsymbol{k})$ are localized, can become of importance at small angles between $\boldsymbol{g}$ vectors. Then the model of independent intersections will overestimate the partial contributions of Bragg planes. In the considered set of $\boldsymbol{g}$ vectors, this can be the case for the (222100) triads. Indeed, the angle between $\boldsymbol{g}$ vectors of the triad is comparatively small, about $19.4^{\circ}$. To remedy the situation, when modeling an optical response of $\mathrm{Zn}-\mathrm{Mg}-R$ quasicrystals, we will treat each triad of the (222100) subset as a single Bragg plane, i. e., we will replace the subset of sixty $\boldsymbol{g}_{222100}$ vectors by twenty effective $\boldsymbol{g}$ vectors, moduli of which are equal to that of $\boldsymbol{g}_{222100}$.

\section{OPTICAL CONDUCTIVITY}

The optical conductivity in metallic and semimetallic solids is determined by the intraband, Drude-type, and the interband transitions of the valence electrons. Both terms of the optical conductivity, $\sigma_{\text {Drude }}(\omega)$ and $\sigma_{\mathrm{ib}}(\omega)$, can be calculated following the Ashcroft and Sturm algorithm, ${ }^{21}$ which was worked out for the usual crystalline metals, and later was successfully applied to an analysis of the fci Al-Cu-Fe optical response. ${ }^{33}$ The Ashcroft and Sturm calculations were carried out in the reduced zone presentation. Since the reduction of electron states to the Brillouin zone is based on the Bloch theorem and, therefore, can not be strictly justified for quasicrystals, we repeated optical conductivity calculations in the extended zone scheme. The main difference in the reduced- and extendedzone presentations is the selection rule for optical transitions. Within the reduced zone scheme, the transitions are vertical, $\boldsymbol{k}^{\prime}=\boldsymbol{k}$, whereas within the extended zone presentation the transitions are indirect-the final quasimomentum is shifted by the reciprocal lattice vector, $\boldsymbol{k}^{\prime}=\boldsymbol{k}-\boldsymbol{g}$. Nevertheless, as can be expected, calculations in both presentations lead to the same final results for $\sigma(\omega)$.

The Ashcroft and Sturm $\sigma(\omega)$ formulas were derived for polyvalent metals, the Fermi level in which is positioned above a pseudogap. Here we will supplement the Ashcroft and Sturm results by formulas appropriate to the cases when the Fermi level is positioned within a pseudogap and below it. The final $\sigma_{\mathrm{ib}}(\omega)$ and $\sigma_{\text {Drude }}(\omega)$ formulas are presented in the following subsections, and a scheme of their derivation is outlined in Appendix.

\section{A. Interband optical conductivity}

Within the framework of the independent intersections model, the interband optical conductivity can be represented by the following compact formula:

$$
\begin{gathered}
\sigma_{\mathrm{ib}}(\omega)=\sum_{g \in \mathcal{G}} \frac{e^{2} g}{24 \pi \hbar} \int_{1}^{x_{1}} \frac{d x S(x)}{x^{3} \sqrt{x^{2}-1}} K(x, z, b), \\
K(x, z, b)=\frac{z}{i \pi}\left[\frac{1}{x-(z+i b)}+\frac{1}{x+(z+i b)}\right] .
\end{gathered}
$$

Here the dimensionless integration variable $x$ is related to $\gamma$ in Eq. (9) as $x=\left(\gamma^{2}+1\right)^{1 / 2}$. The dimensionless parameters $z=\hbar \omega / \Delta_{g}$ and $b=\Gamma / \Delta_{g}$ of the kernel $K(x, z, b)$ correspond to the photon energy $\hbar \omega$ and the broadening parameter $\Gamma$, respectively. The $S(x)$ function acquires different expressions depending on the relative position of the Fermi level with respect to a pseudogap:

(i) The Fermi level below a pseudogap,

$$
S(x)= \begin{cases}0, & 1<x<x_{0}, \\ \frac{\left(x-x_{0}\right)\left(x_{1}-x\right)}{2\left(x_{1}+x_{0}\right)}, & x_{0}<x<x_{1} .\end{cases}
$$

(ii) The Fermi level within a pseudogap,

$$
S(x)=\frac{\left(x-x_{0}\right)\left(x_{1}-x\right)}{2\left(x_{1}+x_{0}\right)}, \quad 1<x<x_{1} .
$$

(iii) The Fermi level above a pseudogap,

$$
S(x)= \begin{cases}x, & 1<x<\left|x_{0}\right|, \\ \frac{\left(x-x_{0}\right)\left(x_{1}-x\right)}{2\left(x_{1}+x_{0}\right)}, & \left|x_{0}\right|<x<x_{1} .\end{cases}
$$

Here the dimensionless energies $x_{0}$ and $x_{1}$ are defined as

$$
\begin{aligned}
& x_{0}=\frac{4 \varepsilon_{0}}{\Delta_{g}}\left[1-\sqrt{\frac{\varepsilon_{\mathrm{F}}}{\varepsilon_{0}}+\left(\frac{\Delta_{g}}{4 \varepsilon_{0}}\right)^{2}}\right], \\
& x_{1}=\frac{4 \varepsilon_{0}}{\Delta_{g}}\left[1+\sqrt{\frac{\varepsilon_{\mathrm{F}}}{\varepsilon_{0}}+\left(\frac{\Delta_{g}}{4 \varepsilon_{0}}\right)^{2}}\right] .
\end{aligned}
$$

\section{B. Intraband optical conductivity}

The intraband optical conductivity has the Drude-type dispersion

$$
\sigma_{\text {Drude }}(\omega)=\frac{\sigma_{\mathrm{dc}}}{1-i \omega \tau}
$$

where $\sigma_{\mathrm{dc}}$ is the static electric conductivity and $\tau$ is the relaxation time.

The deviations of the electron energy spectrum from the free electron parabola affect the group velocity of electrons and, consequently, reduce the static conductivity. The effect is accounted for by the optical mass $m_{\mathrm{opt}}$, which enters the static conductivity formula $\sigma_{\mathrm{dc}}=e^{2} n \tau / m_{\mathrm{opt}}$. Within the model of independent intersections, the optical mass is given by the formula

$$
\frac{1}{m_{\mathrm{opt}}}=\frac{1}{m_{0}}\left[1-\sum_{\boldsymbol{g} \in \mathcal{G}} \frac{m_{0} g \Delta_{\boldsymbol{g}}}{24 \pi \hbar^{2} n} C\right]
$$




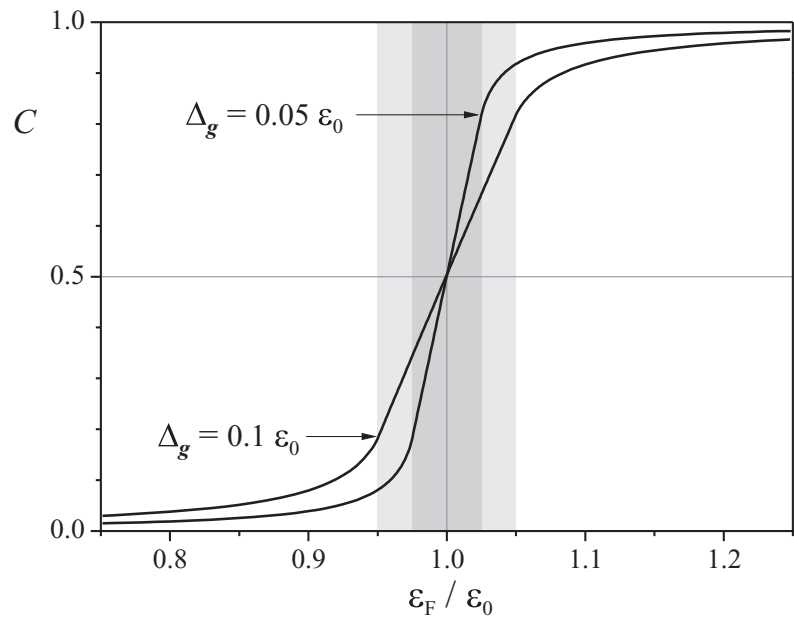

FIG. 7. Dependence of the $C$ coefficient on the position of the Fermi level with respect to a pseudogap ( $\varepsilon_{0}$ is the intersection energy, $\left.\varepsilon_{0}=\hbar^{2}(g / 2)^{2} / 2 m_{0}\right)$.

and the intraband optical conductivity can be represented in the form

$$
\sigma_{\text {Drude }}(\omega)=\frac{1}{1-i \omega \tau}\left[\frac{\omega_{\mathrm{p}}^{2} \tau}{4 \pi}-\sum_{\boldsymbol{g} \in \mathcal{G}} \frac{e^{2} g}{24 \pi \hbar} \frac{\tau \Delta_{\boldsymbol{g}}}{\hbar} C\right],
$$

where $\omega_{\mathrm{p}}=\sqrt{4 \pi e^{2} n / m_{0}}$ is the plasma frequency. The dimensionless coefficient $C$ in formulas (21) and (22) is defined by the expression

$$
C=\frac{2}{\pi} \int_{1}^{x_{1}} \frac{d x S(x)}{x^{2} \sqrt{x^{2}-1}},
$$

where $S(x)$ function is determined by (15)-(17).

The $C$ coefficient depends on the position of the Fermi level with respect to a pseudogap and on a pseudogap value. The $C=C\left(\varepsilon_{\mathrm{F}} / \varepsilon_{0}\right)$ function at several given $\Delta_{g} / \varepsilon_{0}$ values is presented graphically in Fig. 7. As seen, a contribution of pseudogaps to the intraband conductivity decreases when they are raised above the Fermi level.

The $C$ coefficient determines the dependence of both intraband and interband optical conductivity on the position of the Fermi level with respect to a pseudogap. Indeed, the spectral weight of the interband transitions, $\int_{0}^{\infty} d \omega \operatorname{Re} \sigma_{\mathrm{ib}}(\omega)$, as can be easily proved by a straightforward integration of the $K$-kernel (14), is given by the formula

$$
W_{\mathrm{ib}}=\int_{0}^{\infty} d \omega \operatorname{Re} \sigma_{\mathrm{ib}}(\omega)=\sum_{g \in \mathcal{G}} \frac{e^{2} g \Delta_{g}}{48 \hbar^{2}} C .
$$

As it can be seen, the intensity of the interband absorption peaks is proportional to the pseudogap width $\Delta_{g}$ and to the $C$ coefficient.

The spectral weight of the Drude peak is

$$
W_{\text {Drude }}=\int_{0}^{\infty} d \omega \operatorname{Re} \sigma_{\text {Drude }}(\omega)=\frac{\omega_{\mathrm{p}}^{2}}{8}-\sum_{\boldsymbol{g} \in \mathcal{G}} \frac{e^{2} g \Delta_{g}}{48 \hbar^{2}} C .
$$

As seen from (24) and (25), the spectral weight of the interband absorption is compensated by a reduction of the Drude peak by exactly the same amount. This ensures the total spectral weight of optical conductivity to satisfy the oscillator sum rule,

$$
W=\int_{0}^{\infty} d \omega \operatorname{Re} \sigma(\omega)=\frac{\omega_{\mathrm{p}}^{2}}{8} .
$$

\section{Limiting cases}

The integrals in formulas (13) and (23) of the interband conductivity and the $C$ coefficient can be calculated analytically. However, the resulting algebraic expressions are lengthy and complicated, and we will not present them here.

The transparent formulas for the interband conductivity can be obtained in the limiting cases of weak and strong scattering.

\section{Weak scattering limit}

In the limit of weak scattering, when $\Gamma \ll \Delta_{g}$, the $K$ kernel (14) reduces to the form

$$
K(x, z, b)=\frac{z}{i \pi}\left[\frac{1}{x-z}+i \pi \delta(x-z)+\frac{1}{x+z}+i \pi \delta(x+z)\right],
$$

and the real part of the interband conductivity is easily calculated due to a simple integration with the $\delta(x-z)$ function. The interband absorption line/band due to a single Bragg plane acquires the form

$$
\operatorname{Re} \sigma_{\mathrm{ib}}^{g}(\omega)=\frac{e^{2} g}{24 \pi \hbar}\left(\frac{\Delta_{g}}{\hbar \omega}\right)^{2} \frac{\left.S(x)\right|_{x=\hbar \omega / \Delta_{g}}}{\sqrt{\left(\hbar \omega / \Delta_{g}\right)^{2}-1}} .
$$

When the Fermi level is below a pseudogap, the $S(x)$ function is given by (15), and formula (28) corresponds to the well-known Butcher result, ${ }^{34}$ widely used in an analysis of the optical conductivity of alkali metals. When the Fermi level is above a pseudogap, the $S(x)$ function is given by (17), and formula (28) repeats the Ashcroft and Sturm ${ }^{21}$ and Golovashkin et al. ${ }^{35}$ results, derived for polyvalent metals.

When the Fermi level is positioned within a pseudogap, $\varepsilon_{0}-\frac{1}{2} \Delta_{g}<\varepsilon_{\mathrm{F}}<\varepsilon_{0}+\frac{1}{2} \Delta_{g}$, the $S(x)$ function is given by (16), and formula (28) predicts an interband absorption band with the absorption edge determined by the pseudogap width $\Delta_{g}$. The absorption onset has the $\operatorname{Re} \sigma_{\mathrm{ib}}^{g}(\omega) \propto 1 / \sqrt{\hbar \omega-\Delta_{g}}$ singularity, which is smeared out at finite values of the broadening parameter (Fig. 8).

\section{Strong scattering limit}

In the limit of strong scattering (considered by Burkov et al.), ${ }^{33}$ when $\Gamma \gg \Delta_{g}$, the $K$ kernel (14) reduces to the form

$$
K(x, z, b)=\frac{i 2 x z}{\pi(z+i b)^{2}} .
$$

The interband optical conductivity due to a single Bragg plane in this limit is given by the formula

$$
\sigma_{\mathrm{ib}}^{g}(\omega)=\frac{e^{2} g}{24 \pi \hbar} \frac{\Delta_{g}}{\Gamma} C \frac{i \Gamma \hbar \omega}{(\hbar \omega+i \Gamma)^{2}} .
$$

The position of the absorption band is now determined by the broadening parameter $\Gamma$.

The strong scattering limit, $\Gamma \gg \Delta_{g}$, is relevant for very narrow pseudogaps, which in quasicrystals correspond to the reciprocal lattice nodes with low structure factors $S_{g}$. When modeling optical conductivity of $\mathrm{Zn}-\mathrm{Mg}-R$ quasicrystals, we 


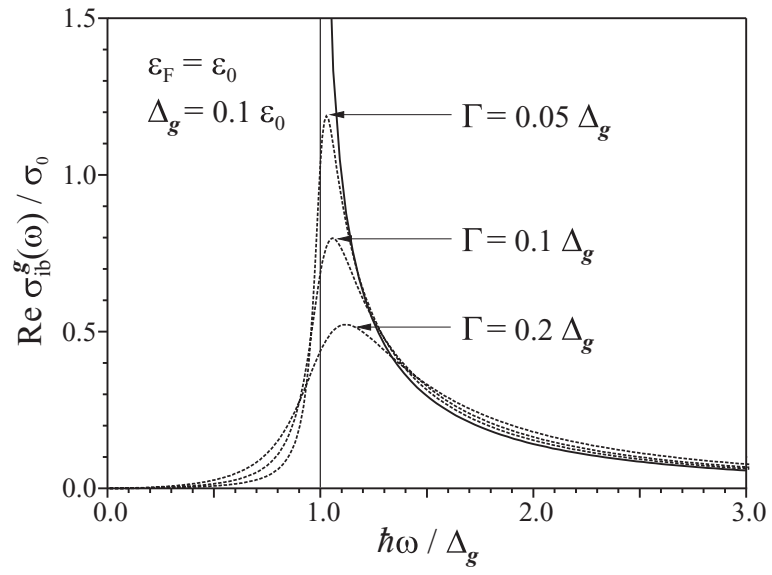

FIG. 8. The interband absorption band when the Fermi level is positioned within a pseudogap. Full curve corresponds to Eq. (28), while dashed curves [Eq. (13)] show the band at finite values of the broadening parameter $\Gamma$. The optical conductivity is presented in $\sigma_{0}=e^{2} g / 24 \pi \hbar$ units.

will use formula (30) to account for a possible contribution of the low- $S_{g}$ pseudopotentials, which correspond to $g \notin \mathcal{G}$ vectors. Another anticipated effect of the low- $S_{g}$ pseudopotentials on optical spectra, which was not dealt with in the present study, is a broadening of the main, $\mathcal{G}$-induced, optical features by the low- $S_{g}$ scattering.

\section{ANALYSIS OF Zn-Mg-R OPTICAL SPECTRA}

The experimental optical conductivity $\sigma(\omega)$ spectra of Zn$\mathrm{Mg}-R$ quasicrystals are presented by dots in Fig. 9. All three, $\mathrm{Zn}-\mathrm{Mg}-\mathrm{Y}, \mathrm{Zn}-\mathrm{Mg}-\mathrm{Ho}$, and $\mathrm{Zn}-\mathrm{Mg}-\mathrm{Er}$, quasicrystals manifest a similar dispersion-the spectra exhibit a distinct optical feature at about $1 \mathrm{eV}$, which obviously is due to the interband transitions, and the intraband Drude-type contribution at low frequencies. A small kink in the $\mathrm{Zn}-\mathrm{Mg}-\mathrm{Ho}$ and $\mathrm{Zn}-\mathrm{Mg}-\mathrm{Er}$ spectra at about $0.13 \mathrm{eV}$ is an artefact. It corresponds to a division line between data recorded by FIR reflection spectroscopy and IR spectroscopic ellipsometry techniques.

The theoretical simulations of the $\sigma(\omega)$ spectra, based on the formulas presented in the previous section, can easily be carried out at given values of the Fermi energy $\varepsilon_{\mathrm{F}}$, the pseudogap widths $\Delta_{222100}$ and $\Delta_{311111}$, and the broadening parameters.

Although the dominant role in an optical response of $\mathrm{Zn}-\mathrm{Mg}-R$, as well as of other metals, is played by the valence electrons, one can expect a small possible contribution of the bound $\mathrm{Zn} 3 d$ core electrons. Since the $\mathrm{Zn} 3 d$ level is at the bottom of the valence band, approximately $10 \mathrm{eV}$ below the Fermi level, ${ }^{16,17}$ a feasible $\mathrm{Zn} 3 d$ polarization was taken into account by introducing the high-frequency dielectric constant into the QC dielectric function

$$
\varepsilon(\omega)=\varepsilon_{\infty}+i \frac{4 \pi}{\omega}\left[\sigma_{\text {Drude }}(\omega)+\sigma_{\mathrm{ib}}(\omega)\right] .
$$

Results of the Zn-Mg- $R$ optical conductivity calculations are presented by curves in Fig. 9. As seen, the theory nicely reproduces the experimental data.
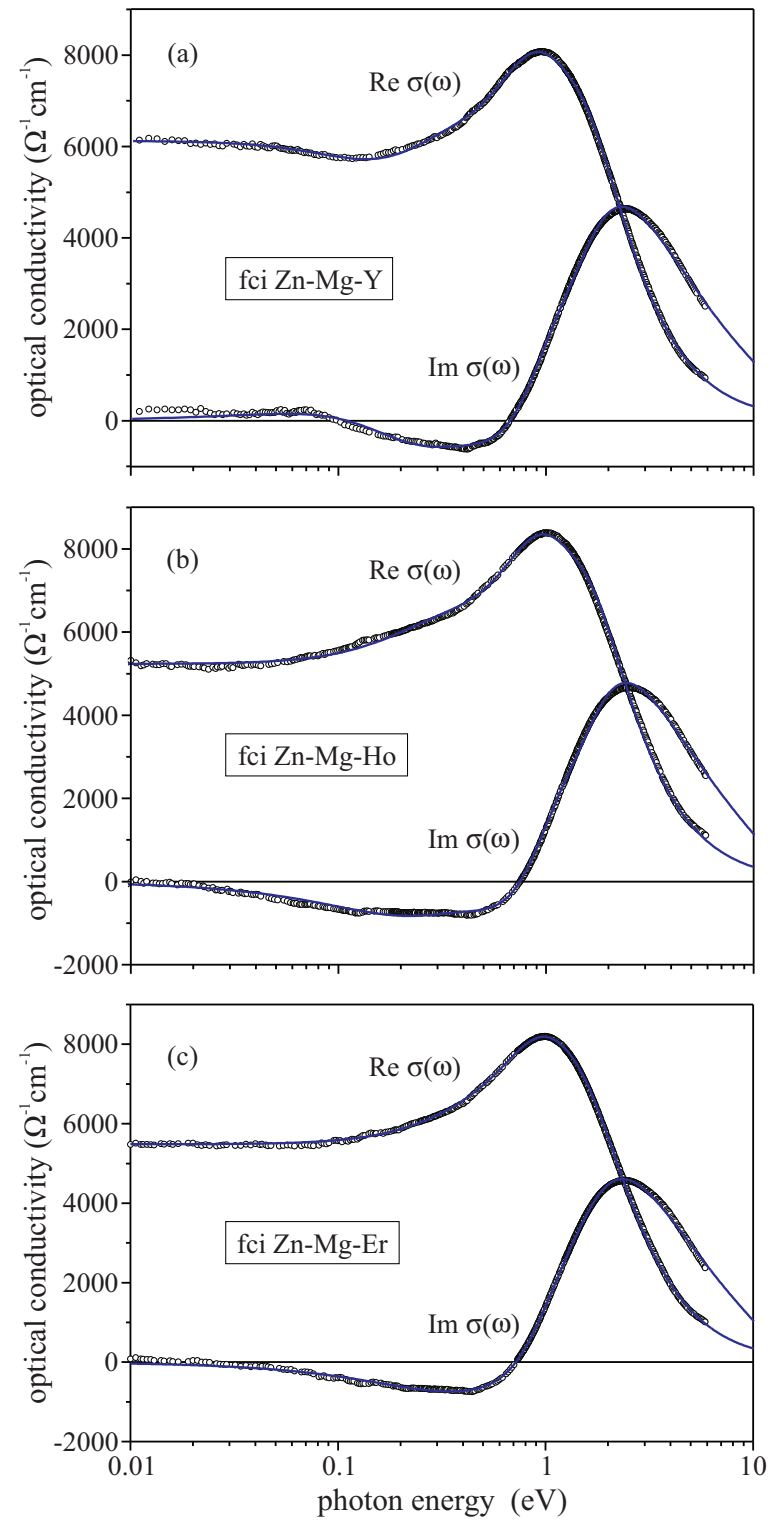

FIG. 9. (Color online) The optical conductivity spectra of (a) Zn$\mathrm{Mg}-\mathrm{Y}$, (b) Zn-Mg-Ho, and (c) Zn-Mg-Er quasicrystals. Dots present experimental data. Curves correspond to theoretical calculations.

The high-frequency dielectric constant, which was treated as a fitting parameter, is, as expected, close to unity, $\varepsilon_{\infty}=$ 1.35, 1.56, and 1.58 for $\mathrm{Zn}-\mathrm{Mg}-\mathrm{Y}, \mathrm{Zn}-\mathrm{Mg}-\mathrm{Ho}$, and $\mathrm{Zn}-\mathrm{Mg}$ $\mathrm{Er}$, respectively, and does not essentially influence an optical response of the quasicrystals.

The plasma frequency $\omega_{\mathrm{p}}$ values, which determine the total spectral weight of $\operatorname{Re} \sigma(\omega)$ spectra [see Eq. (26)], are of about 11.5-11.9 eV (Table II) and are close to the theoretical values $\omega_{\mathrm{p}}^{\mathrm{Zn}-\mathrm{Mg}-\mathrm{Y}}=12.7 \mathrm{eV}, \omega_{\mathrm{p}}^{\mathrm{Zn}-\mathrm{Mg}-\mathrm{Ho}}=12.5 \mathrm{eV}$, and $\omega_{\mathrm{p}}^{\mathrm{Zn}-\mathrm{Mg}-\mathrm{Er}}=12.4 \mathrm{eV}$, predicted by the formula $\omega_{\mathrm{p}}=$ $\sqrt{4 \pi e^{2} n / m_{0}}$ at the valence electron concentration $n$ (Table I), which was determined from the mass density and the average valence values $\bar{Z}_{\mathrm{Zn}-\mathrm{Mg}-\mathrm{Y}}=2.09, \bar{Z}_{\mathrm{Zn}-\mathrm{Mg}-\mathrm{Ho}}=2.10$, and $\bar{Z}_{\mathrm{Zn}-\mathrm{Mg}-\mathrm{Er}}=2.11$.

Figure 10 shows the intraband and interband contributions, $\sigma_{\text {Drude }}(\omega)$ and $\sigma_{\mathrm{ib}}(\omega)$, to the $\mathrm{Zn}-\mathrm{Mg}-\mathrm{Y}$ total optical conductivity 
TABLE II. Parameters of the Zn-Mg- $R$ intraband Drude optical conductivity.

\begin{tabular}{lccc}
\hline \hline & Zn-Mg-Y & Zn-Mg-Ho & Zn-Mg-Er \\
\hline$\omega_{\mathrm{p}}(\mathrm{eV})$ & 11.5 & 11.9 & 11.7 \\
$\sigma_{\mathrm{dc}}\left(\Omega^{-1} \mathrm{~cm}^{-1}\right)$ & 6100 & 5200 & 5500 \\
$\hbar / \tau(\mathrm{eV})$ & 0.18 & 0.45 & 0.47 \\
$w_{\text {Drude }}$ & 0.06 & 0.12 & 0.14 \\
$m_{\text {opt }}\left(m_{0}\right)$ & 16 & 8.1 & 7.2 \\
\hline \hline
\end{tabular}

spectrum. The relative spectral weight of the intraband Drude conductivity, $w_{\text {Drude }}=W_{\text {Drude }} / W$, with respect to the total $\sigma(\omega)$ spectral weight $W$, is of about $10 \%$ (see Table II). The relative spectral weight $w_{\text {Drude }}$, as can easily be checked from formulas (21) and (25), unambiguously determines the optical mass, $m_{\text {opt }}=m_{0} / w_{\text {Drude }}$, which, therefore, in the fci $\mathrm{Zn}-\mathrm{Mg}-R$ quasicrystals is approximately $10 m_{0}$.

The parameters of the intraband optical conductivity, the static conductivity and the relaxation time, are presented in Table II. The determined static conductivity values $\sigma_{\mathrm{dc}} \sim$ 5000-6000 $\Omega^{-1} \mathrm{~cm}^{-1}$ roughly correspond to the Fisher et al. results of the electric resistivity measurements: ${ }^{36,37} \sigma_{\mathrm{dc}} \approx$ 6620, 5410, and $5880 \Omega^{-1} \mathrm{~cm}^{-1}$ for $\mathrm{Zn}-\mathrm{Mg}-\mathrm{Y}$, Zn-Mg$\mathrm{Ho}$, and $\mathrm{Zn}-\mathrm{Mg}$-Er, respectively. The relaxation times $\tau \sim$ $0.14-0.410^{-14} \mathrm{~s}$ determined in the present study are longer than the $\tau$ values we reported previously, ${ }^{20}$ where they were deduced from the $\sigma(\omega)$ analysis in a narrower spectral range of
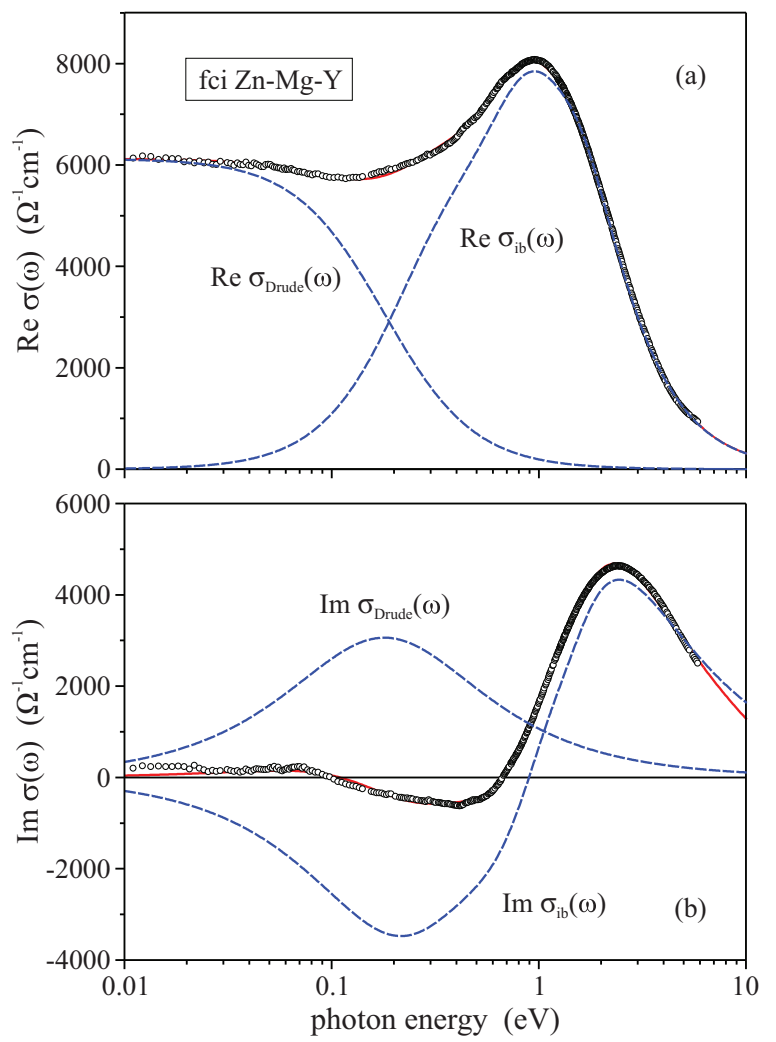

FIG. 10. (Color online) The intraband and interband contributions, $\sigma_{\text {Drude }}(\omega)$ and $\sigma_{\text {ib }}(\omega)$, to the $\mathrm{Zn}-\mathrm{Mg}-\mathrm{Y}$ total optical conductivity spectrum.
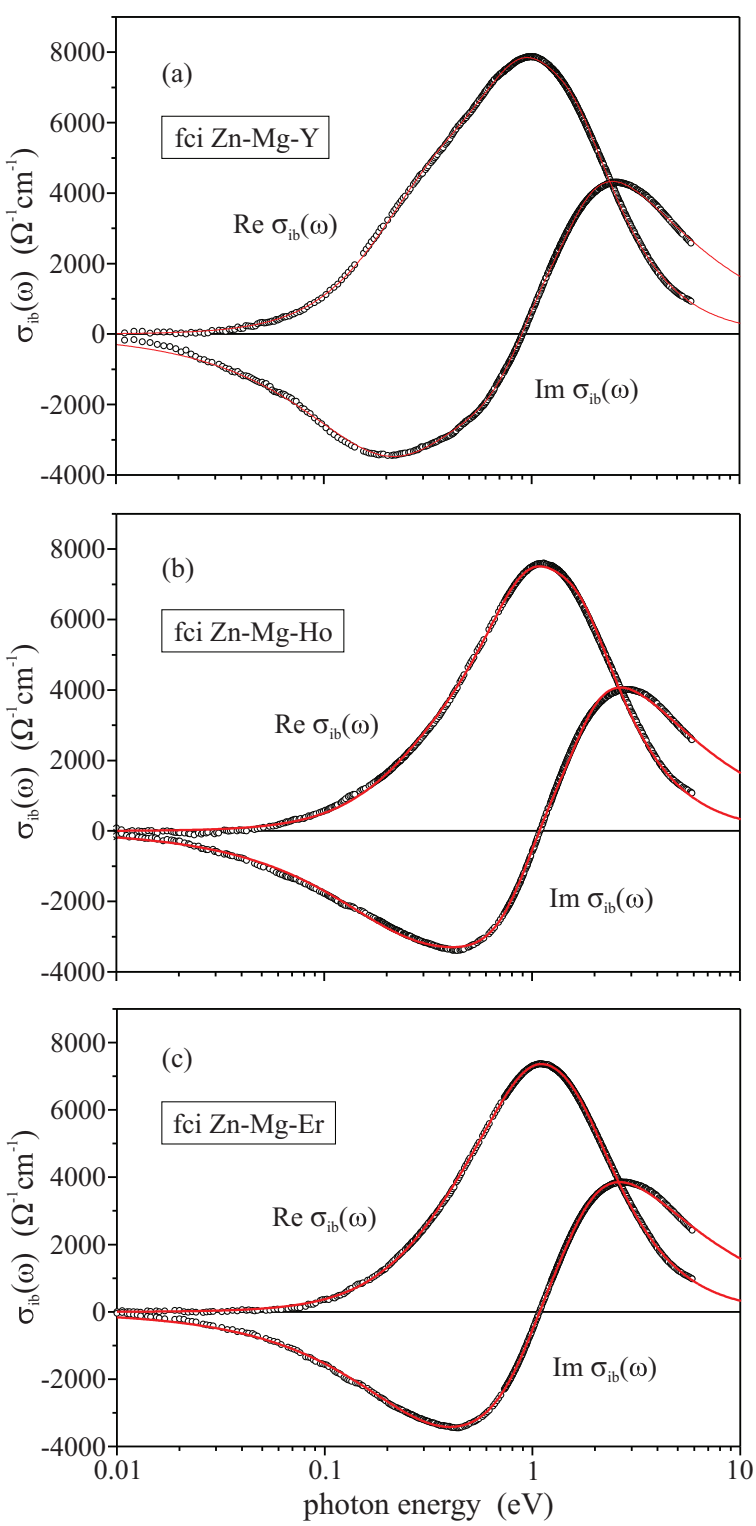

FIG. 11. (Color online) Interband optical conductivity of (a) ZnMg-Y, (b) Zn-Mg-Ho, and (c) Zn-Mg-Er quasicrystals.

0.1-6 eV, but closer to the $\mathrm{Zn}-\mathrm{Mg}-\mathrm{Y}$ and $\mathrm{Zn}-\mathrm{Mg}-\mathrm{Tb}$ relaxation times reported by Chernikov et al., ${ }^{15} \tau \sim 0.510^{-14} \mathrm{~s}$.

The $\mathrm{Zn}-\mathrm{Mg}-R$ interband optical conductivity $\sigma_{\mathrm{ib}}(\omega)$ spectra, which were obtained by a subtraction of the determined Drude contribution $\sigma_{\text {Drude }}(\omega)$ from the total optical conductivity, are presented in Fig. 11, where dots and curves correspond to experimental data and theoretical calculations, respectively. The interband optical conductivity is due to the optical transitions across the 222100 and 311111 pseudogaps and due to a contribution of the $g \notin \mathcal{G}$ pseudopotentials with low structure factors. The relative spectral weights of $\operatorname{Re} \sigma_{\mathrm{ib}, 222100}(\omega)$, $\operatorname{Re} \sigma_{\mathrm{ib}, 311111}(\omega)$, and $\operatorname{Re} \sigma_{\mathrm{ib}, g \notin \mathcal{G}}(\omega)$ interband conductivities (with respect to the total spectral weight $W$ ) are around $0.5,0.3$, and 0.1 , respectively (see Table III). The partial contributions to the $\mathrm{Zn}-\mathrm{Mg}-\mathrm{Y}$ interband optical conductivity spectrum are shown in Fig. 12.

The parameters of the electron energy spectrum, the Fermi energy $\varepsilon_{\mathrm{F}}$, the pseudogap widths $\Delta_{222100}$ and $\Delta_{311111}$, 
TABLE III. The relative spectral weights of the interband transitions across the 222100 and 311111 pseudogaps and of the transitions induced by the low $S_{g}$-factor pseudopotentials.

\begin{tabular}{lccc}
\hline \hline & Zn-Mg-Y & Zn-Mg-Ho & Zn-Mg-Er \\
\hline$w_{222100}$ & 0.51 & 0.50 & 0.49 \\
$w_{311111}$ & 0.29 & 0.29 & 0.25 \\
$w_{g \notin \mathcal{G}}$ & 0.14 & 0.08 & 0.12 \\
\hline \hline
\end{tabular}

and the broadening parameters, which were determined by standard least-squares technique, are presented in Table IV. The Fermi energy $\varepsilon_{\mathrm{F}}=9.20-9.25 \mathrm{eV}$ and the pseudogap $\Delta_{222100}=0.55-0.61 \mathrm{eV}$ and $\Delta_{31111}=1.34-1.45 \mathrm{eV}$ values are rather close to those, deduced from an analysis of the $\mathrm{Zn}-\mathrm{Mg}-R$ valence band photoemission spectra, ${ }^{16,17}$ namely, 9.31-9.32 eV for the Fermi energy, and 0.61-0.64 eV and $0.98-1.25 \mathrm{eV}$ for the $\Delta_{222100}$ and $\Delta_{31111}$ pseudogaps. This is a sound support for a self-consistency of the QC electron energy spectrum model suggested-it nicely reproduces experimental data for both the optical and the PE response of the $\mathrm{Zn}-\mathrm{Mg}-R$ quasicrystals at actually the same values of energy spectrum parameters.

The broadening parameter $\Gamma_{\mathcal{G}}$ of the interband transitions across the pseudogaps, which was assumed to be the same for $\Delta_{222100}$ and $\Delta_{311111}$, is of about $0.62-0.68 \mathrm{eV}$. It differs from the broadening parameter determined in PE analysis, $\Gamma_{\mathrm{PE}} \approx 0.22-0.24 \mathrm{eV}$. We do not know the exact reasons for the difference. Here it should be mentioned that the
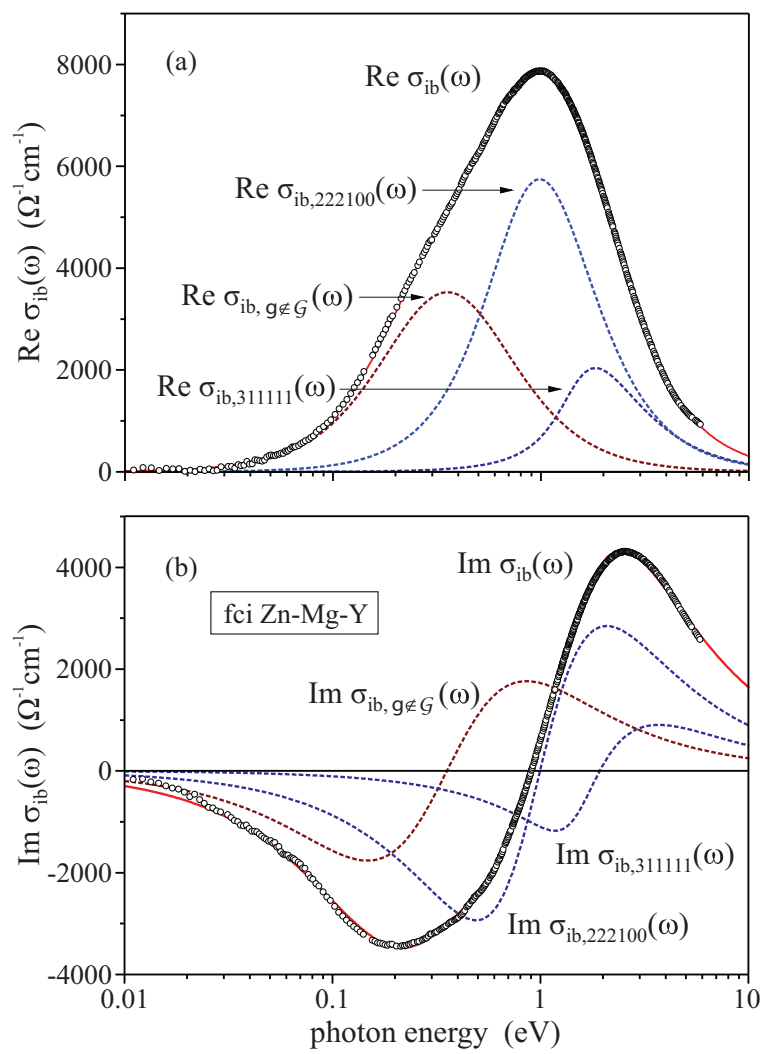

FIG. 12. (Color online) The partial contributions $\sigma_{\mathrm{ib}, 222100}(\omega)$, $\sigma_{\mathrm{ib}, 311111}(\omega)$, and $\sigma_{\mathrm{ib}, g \notin \mathcal{G}}(\omega)$ to the $\mathrm{Zn}-\mathrm{Mg}-\mathrm{Y}$ interband optical conductivity spectrum.
TABLE IV. Parameters of fci Zn-Mg- $R$ electron energy spectrum: the Fermi energy $\varepsilon_{F}$, the pseudogaps $\Delta_{222100}$ and $\Delta_{31111}$, and the broadening parameters $\Gamma_{222100}=\Gamma_{311111} \equiv \Gamma_{\mathcal{G}}$ and $\Gamma_{g \notin \mathcal{G}}$. The values of the parameters, deduced from an analysis of the photoemission data, ${ }^{16,17}$ are presented in parentheses.

\begin{tabular}{lccc}
\hline \hline & Zn-Mg-Y & Zn-Mg-Ho & Zn-Mg-Er \\
\hline$\varepsilon_{\mathrm{F}}(\mathrm{eV})$ & $9.25(9.31)$ & $9.25(9.32)$ & $9.20(9.32)$ \\
$\Delta_{222100}(\mathrm{eV})$ & $0.55(0.63)$ & $0.60(0.61)$ & $0.61(0.64)$ \\
$\Delta_{31111}(\mathrm{eV})$ & $1.36(1.16)$ & $1.45(0.98)$ & $1.34(1.25)$ \\
$\Gamma_{\mathcal{G}}(\mathrm{eV})$ & $0.62(0.23)$ & $0.63(0.22)$ & $0.68(0.24)$ \\
$\Delta_{g \notin \mathcal{G}}(\mathrm{eV})$ & $\ll \Gamma_{g \notin \mathcal{G}}$ & $\ll \Gamma_{g \notin \mathcal{G}}$ & $\ll \Gamma_{g \notin \mathcal{G}}$ \\
$\Gamma_{g \notin \mathcal{G}}(\mathrm{eV})$ & 0.35 & 0.41 & 0.55 \\
\hline \hline
\end{tabular}

broadening in the optical and PE data analysis is introduced by different phenomenological schemes: in optics, as a Lorentzian-type broadening of the spectral lines [see the $K$-kernel formula (14)], and in PE, as a Gaussian convolution of the density of states.

The broadening parameter $\Gamma_{g \notin \mathcal{G}}$ of the interband transitions induced by the pseudopotentials with low structure factors is about $0.35-0.55 \mathrm{eV}$ (Table IV). Since the line shape of $\sigma_{\mathrm{ib}, g \notin \mathcal{G}}(\omega)$ is determined solely by the broadening parameter [see Eq. (30)], the pseudopotentials $V_{g \notin \mathcal{G}}$ and the corresponding pseudogaps $\Delta_{g \notin \mathcal{G}}=2\left|V_{g \notin \mathcal{G}}\right|$ can not be directly deduced from the optical spectra analysis. They determine the spectral weight of the $\{\boldsymbol{g} \notin \mathcal{G}\}$-transitions,

$$
W_{g \notin \mathcal{G}}=\sum_{g \notin \mathcal{G}} \frac{e^{2} g \Delta_{g \notin \mathcal{G}}}{48 \hbar^{2}} C .
$$

A rough estimate of the $\Delta_{g \notin \mathcal{G}}$ pseudogaps can be obtained assuming that all $\boldsymbol{g}$ vectors contributing to the sum in Eq. (32) have the same moduli, $g \approx 2 k_{\mathrm{F}}$, and correspond to the same pseudogap values. Then, under assumption that a multiplicity of the $g \notin \mathcal{G}$ vectors is 60 and that $C \approx 1$, we obtain an estimate of $\Delta_{g \notin \mathcal{G}} \sim 0.03-0.04 \mathrm{eV}$. Such low values of $\Delta_{g \notin \mathcal{G}}$ pseudogaps seem to be physically acceptable taking into account that, in the $\mathrm{Zn}-\mathrm{Mg}-\mathrm{R}$ high-resolution diffraction patterns, the Bragg peaks with their intensity ratios below $\sim 10^{-3}$ manifest themselves. ${ }^{38}$

Finally we would like to point out that, due to a rather smooth spectral shape of the experimental $\mathrm{Zn}-\mathrm{Mg}-R$ optical spectra, a determination of both intraband, and interband parameters, most probably, is not unambiguous. Previously we qualitatively explained the experimental $\mathrm{Zn}-\mathrm{Mg}-R$ optical conductivity spectra, recorded in the narrower spectral range of $0.1-6 \mathrm{eV}$, by optical transitions across a single 311111 pseudogap. ${ }^{20}$ However, this required very short Drude relaxation times, $\tau \sim 0.0310^{-14} \mathrm{~s}(\hbar / \tau \sim 2 \mathrm{eV})$, a decrease of the $\Delta_{311111}$ value down to $\sim 0.8 \mathrm{eV}$, and an increase of the Fermi energy up to $9.7-9.8 \mathrm{eV}$. The reliability of the set of parameters, determined in the present study, we suppose, is confirmed by the fact that the electron energy spectrum in the vicinity of the Fermi level is predicted to have the same structure as that predicted from the photoemission data analysis (Fig. 13) - the Fermi level is slightly above the 222100 pseudogap, and is positioned in a lower part of the 311111 pseudogap. 


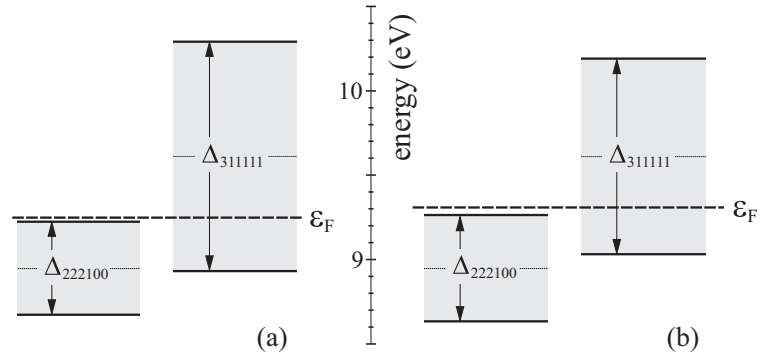

FIG. 13. The Zn-Mg-Y electron energy spectrum in the vicinity of the Fermi level, predicted from analysis of optical (a) and photoemission spectroscopy (b) data.

\section{SUMMARY}

Summarizing, we presented the $\mathrm{Zn}-\mathrm{Mg}-R(R=\mathrm{Y}$, Ho, Er) optical conductivity spectra, recorded in the $0.01-6 \mathrm{eV}$ spectral range by a combined spectroscopic ellipsometry and FIR reflection spectroscopy technique. The model of $\mathrm{Zn}-\mathrm{Mg}-R$ electron energy spectrum, suggested previously in an analysis of the valence-band photoemission spectra, ${ }^{16}$ was developeda hypothetical band structure of the quasicrystals in the vicinity of the Fermi level was calculated and the NFE model of independent intersections was formulated in the extended zone presentation. The Ashcroft and Sturm scheme ${ }^{21}$ of the optical conductivity theoretical calculations was extended to account for various positions of the Fermi level with respect to a pseudogap.

An analysis of the Zn-Mg- $R$ optical conductivity spectra shows that the intraband, Drude-type, optical transitions contribute to the total optical conductivity with a relative spectral weight of about $10 \%$. The Drude relaxation times are about $0.14-0.410^{-14} \mathrm{~s}$.

The interband optical conductivity is predominantly due to the optical transitions across 222100 and 311111 pseudogaps. Their relative spectral weight is about $80 \%$. The relative spectral weight of an additional contribution due to the pseudopotentials, corresponding to the reciprocal lattice nodes with low structure factors, is about $10 \%$.

The experimental $\mathrm{Zn}-\mathrm{Mg}-R$ optical conductivity spectra are reproduced in detail by theoretical calculations performed within the framework of the suggested electron energy spectrum model. The set of electron energy spectrum parameters determined from an analysis of the optical data predicts actually the same Fermi-level vicinity electron energy spectrum structure as was previously predicted from an analysis of photoemission data. ${ }^{16,17}$

\section{ACKNOWLEDGMENTS}

The authors gratefully acknowledge support by the Research Council of Lithuania (Grant No. MIP-081/2012). The Knut and Alice Wallenberg foundation is acknowledged for support to instrumentation.

\section{APPENDIX: DERIVATION OF $\sigma(\omega)$ FORMULAS}

\section{Interband optical conductivity}

In the extended zone presentation, the general formula of the interband optical conductivity ${ }^{39,40}$ for the optically isotropic system has the form

$$
\sigma_{\mathrm{ib}}(\omega)=\frac{2 e^{2} \hbar^{2} \omega}{i 3 m_{0} \Omega} \sum_{\boldsymbol{k} \neq \boldsymbol{k}^{\prime}} \frac{f_{\boldsymbol{k}} f_{\boldsymbol{k}^{\prime} \boldsymbol{k}}}{\left[\varepsilon\left(\boldsymbol{k}^{\prime}\right)-\varepsilon(\boldsymbol{k})\right]^{2}-(\hbar \omega+i \Gamma)^{2}},
$$

where $f_{\boldsymbol{k}}$ is the Fermi distribution function and $f_{\boldsymbol{k}^{\prime} \boldsymbol{k}}$ is the oscillator strength

$$
f_{\boldsymbol{k}^{\prime} \boldsymbol{k}}=\frac{2}{m_{0}\left[\varepsilon\left(\boldsymbol{k}^{\prime}\right)-\varepsilon(\boldsymbol{k})\right]}\left|\left\langle\boldsymbol{k}^{\prime}|\boldsymbol{p}| \boldsymbol{k}\right\rangle\right|^{2} .
$$

The straightforward calculations of the oscillator strength within the model of independent intersections, when the electron wavefunctions and energy spectrum are given by formulas (3) and (10)-(12), yield the following answer for the oscillator strength:

$$
f_{\boldsymbol{k}^{\prime} \boldsymbol{k}}=\sum_{\boldsymbol{g} \in \mathcal{G}} \frac{\gamma}{|\gamma|} \frac{4 \varepsilon_{0}}{\Delta_{\boldsymbol{g}}\left(\gamma^{2}+1\right)^{3 / 2}} \delta_{\boldsymbol{k}^{\prime}, \boldsymbol{k}-\boldsymbol{g}}
$$

where $\varepsilon_{0}=\hbar^{2}\left(\frac{1}{2} g\right)^{2} / 2 m_{0}$ is the intersection energy, $\Delta_{g}$ is the pseudogap width, and $\gamma$ is the dimensionless function (9) of the quasimomentum $\boldsymbol{k}$ component $k_{\|}$along the $\boldsymbol{g}$-direction.

Inserting (A3) into (A1), taking a sum over the final states $\boldsymbol{k}^{\prime}$, and, changing a sum over $\boldsymbol{k}$ to an integral, one obtains the intermediate $\sigma_{\mathrm{ib}}(\omega)$ expression

$$
\begin{aligned}
\sigma_{\mathrm{ib}}(\omega)= & \sum_{g \in \mathcal{G}} \frac{e^{2} \hbar^{2} \omega}{i 12 \pi^{3} m_{0}} \int d^{3} k \frac{\gamma}{|\gamma|} \frac{4 \varepsilon_{0}}{\Delta_{g}\left(\gamma^{2}+1\right)^{3 / 2}} \\
& \times \frac{f_{k}}{\left(\Delta_{g} \sqrt{\gamma^{2}+1}\right)^{2}-(\hbar \omega+i \Gamma)^{2}} .
\end{aligned}
$$

Choosing the cylindrical coordinate system for $\boldsymbol{k}$ integration with a polar axis directed along $\boldsymbol{g}$-vector, the formula (A4) can be rewritten as

$$
\begin{aligned}
\sigma_{\mathrm{ib}}(\omega)= & \sum_{g \in \mathcal{G}} \frac{e^{2} \omega}{i 6 \pi^{2}} \int_{-\infty}^{\infty} d k_{\|} \frac{4 \varepsilon_{0}}{\Delta_{g}\left(1+\gamma^{2}\right)^{3 / 2}} \\
& \times \frac{S\left(k_{\|}\right)}{\left(\Delta_{g} \sqrt{1+\gamma^{2}}\right)^{2}-(\hbar \omega+i \Gamma)^{2}},
\end{aligned}
$$

where $S\left(k_{\|}\right)$is the dimensionless function defined as

$$
S\left(k_{\|}\right)=\frac{\gamma}{|\gamma|} \frac{\hbar^{2}}{m_{0}} \int_{0}^{\infty} k_{\perp} d k_{\perp} f_{k} .
$$

As seen from (A5), the interband optical conductivity is a sum of partial contributions of the $g \in \mathcal{G}$ Bragg planes. Each term of the sum is localized in the $\boldsymbol{k}$-space in a vicinity of the corresponding Bragg plane. Therefore, when calculating the $S\left(k_{\|}\right)$-function, the electron energy $\varepsilon(\boldsymbol{k})$, which enters the Fermi distribution, can be replaced by $\varepsilon(\boldsymbol{k})=\epsilon_{\boldsymbol{k}}+\delta \varepsilon_{\boldsymbol{g}}(\boldsymbol{k})$. The final answers for $S\left(k_{\|}\right)$, as a function of the $x \equiv \sqrt{\gamma^{2}+1}$ argument, are presented by formulas (15)-(17). Replacing in (A5) the integration variable to $x=\sqrt{\gamma^{2}+1}$, one reduces the $\sigma_{\mathrm{ib}}(\omega)$ formula to the final form (13). 


\section{Intraband optical conductivity}

The general formula of the intraband optical conductivity $^{39,40}$ for the optically isotropic system has the form

$$
\sigma_{\text {Drude }}(\omega)=\frac{\sigma_{\mathrm{dc}}}{1-i \omega \tau}, \quad \sigma_{\mathrm{dc}}=\frac{e^{2} n \tau}{m_{\mathrm{opt}}},
$$

where the optical mass in the extended zone presentation is determined by the formula

$$
\frac{1}{m_{\mathrm{opt}}}=\frac{1}{4 \pi^{3} n} \int d^{3} k f_{\boldsymbol{k}} \frac{1}{3 \hbar^{2}} \nabla_{\boldsymbol{k}}^{2} \varepsilon(\boldsymbol{k}) .
$$

Here $\nabla_{\boldsymbol{k}}=\partial / \partial \boldsymbol{k}$ is the gradient operator with respect to $\boldsymbol{k}$.

Action of the $\nabla_{k}^{2}$ operator on the energy spectrum (10) yields

$$
\frac{1}{3 \hbar^{2}} \nabla_{\boldsymbol{k}}^{2} \varepsilon(\boldsymbol{k})=\frac{1}{m_{0}}-\frac{1}{3 m_{0}} \sum_{\boldsymbol{g} \in \mathcal{G}} \frac{\gamma}{|\gamma|} \frac{4 \varepsilon_{0}}{\Delta_{\boldsymbol{g}}\left(\gamma^{2}+1\right)^{3 / 2}} .
$$

Inserting (A9) into (A8), one obtains the intermediate expression

$$
\frac{1}{m_{\mathrm{opt}}}=\frac{1}{m_{0}}\left[1-\frac{1}{12 \pi^{3} n} \sum_{g \in \mathcal{G}} \int d^{3} k \frac{\gamma}{|\gamma|} \frac{4 \varepsilon_{0} f_{k}}{\Delta_{\boldsymbol{g}}\left(\gamma^{2}+1\right)^{3 / 2}}\right] .
$$

Choosing the cylindrical coordinate system for $\boldsymbol{k}$-integration, the formula (A10) can be rewritten as

$$
\frac{1}{m_{\mathrm{opt}}}=\frac{1}{m_{0}}\left[1-\frac{m_{0}}{6 \pi^{2} n \hbar^{2}} \sum_{g \in \mathcal{G}} \int_{-\infty}^{\infty} d k_{\|} \frac{4 \varepsilon_{0} S\left(k_{\|}\right)}{\Delta_{g}\left(\gamma^{2}+1\right)^{3 / 2}}\right],
$$

where $S\left(k_{\|}\right)$function is defined by (A6).

Replacing in (A11) the $k_{\|}$-integration variable to $x=$ $\sqrt{\gamma^{2}+1}$, one reduces the optical mass formula to its final form (21).
*Corresponding author: karpus@pfi.lt

${ }^{1}$ J.-M. Frigerio and J. Rivory, J. Non-Cryst. Solids 117/118, 812 (1990).

${ }^{2}$ C. C. Homes, T. Timusk, X. Wu, Z. Altounian, A. Sahnoune, and J. O. Ström-Olsen, Phys. Rev. Lett. 67, 2694 (1991).

${ }^{3}$ V. Demange, A. Milandri, M. C. de Weerd, F. Machizaud, G. Jeandel, and J. M. Dubois, Phys. Rev. B 65, 144205 (2002).

${ }^{4}$ L. Degiorgi, M. A. Chernikov, C. Beeli, and H. R. Ott, Solid State Commun. 87, 721 (1993).

${ }^{5}$ M. A. Chernikov, L. Degiorgi, A. Bernasconi, C. Beeli, and H. R. Ott, Physica B 194-196, 405 (1994).

${ }^{6}$ D. N. Basov, F. S. Pierce, P. Volkov, S. J. Poon, and T. Timusk, Phys. Rev. Lett. 73, 1865 (1994).

${ }^{7}$ H. Werheit, R. Schmechel, K. Kimura, R. Tamura, and T. Lundström, Solid State Commun. 97, 103 (1996).

${ }^{8}$ A. D. Bianchi, F. Bommeli, M. A. Chernikov, U. Gubler, L. Degiorgi, and H. R. Ott, Phys. Rev. B 55, 5730 (1997).

${ }^{9}$ X. Wu, C. C. Homes, S. E. Burkov, T. Timusk, F. S. Pierce, S. J. Poon, S. L. Cooper, and M. A. Karlow, J. Phys.: Condens. Matter 5, 5975 (1993).

${ }^{10}$ D. N. Basov, T. Timusk, F. Barakat, J. Greedan, and B. Grushko, Phys. Rev. Lett. 72, 1937 (1994).

${ }^{11}$ A. D. Bianchi, F. Bommeli, E. Felder, M. Kenzelmann, M. A. Chernikov, L. Degiorgi, H. R. Ott, and K. Edagawa, Phys. Rev. B 58, 3046 (1998).

${ }^{12}$ V. Karpus, G.-J. Babonas, A. Rėza, W. Assmus, and R. Sterzel, Lith. J. Phys. 40, 118 (2000).

${ }^{13}$ A. Suchodolskis, W. Assmus, G.-J. Babonas, L. Giovanelli, U. O. Karlsson, V. Karpus, G. Le Lay, A. Rėza, and E. Uhrig, Acta Phys. Pol. A 107, 412 (2005)

${ }^{14}$ V. Karpus, G.-J. Babonas, A. Rėza, A. Suchodolskis, S. Tumėnas, O. Hunderi, W. Assmus, and S. Brühne, Acta Phys. Pol. A 113, 1005 (2008).

${ }^{15}$ M. A. Chernikov, S. Paschen, E. Felder, P. Vorburger, B. Ruzicka, L. Degiorgi, H. R. Ott, I. R. Fisher, and P. C. Canfield, Phys. Rev. B 62, 262 (2000).
${ }^{16}$ A. Suchodolskis, W. Assmus, L. Giovanelli, U. O. Karlsson, V. Karpus, G. Le Lay, R. Sterzel, and E. Uhrig, Phys. Rev. B 68, 054207 (2003).

${ }^{17}$ A. Suchodolskis, W. Assmus, L. Giovanelli, U. O. Karlsson, V. Karpus, G. Le Lay, and E. Uhrig, J. Phys.: Condens. Matter 16, 9137 (2004).

${ }^{18}$ V. Karpus, A. Suchodolskis, J. Taulavičius, U. O. Karlsson, W. Assmus, S. Brühne, and E. Uhrig, Opt. Mater. 30, 690 (2008).

${ }^{19}$ V. Karpus, G.-J. Babonas, A. Rèza, S. Tumenas, H. Arwin, W. Assmus, and S. Brühne, Z. Kristallogr. 224, 39 (2009).

${ }^{20} \mathrm{~S}$. Tumėnas, V. Karpus, H. Arwin, and W. Assmus, Thin Solid Films 519, 2951 (2011).

${ }^{21}$ N. W. Ashcroft and K. Sturm, Phys. Rev. B 3, 1898 (1971).

${ }^{22}$ A. Langsdorf and W. Assmus, J. Cryst. Growth 192, 152 (1998).

${ }^{23}$ A. Langsdorf and W. Assmus, Cryst. Res. Technol. 34, 261 (1999).

${ }^{24}$ S. Tumėnas, I. Kašalynas, V. Karpus, and H. Arwin, Acta Phys. Pol. A 119, 140 (2011).

${ }^{25}$ T. Fujiwara, in Physical Properties of Quasicrystals, edited by Z. M. Stadnik (Springer, Berlin, 1999), pp. 169-207.

${ }^{26} \mathrm{~J}$. Hafner and M. Krajčí, in Physical Properties of Quasicrystals, edited by Z. M. Stadnik (Springer, Berlin, 1999), pp. 209-256.

${ }^{27}$ Y. Ishii and T. Fujiwara, in Quasicrystals, edited T. Fujiwara and Y. Ishii (Elsevier, The Netherlands, 2008), pp. 171-208.

${ }^{28}$ A. P. Smith and N. W. Ashcroft, Phys. Rev. Lett. 59, 1365 (1987).

${ }^{29}$ T. Fujiwara, S. Yamamoto, and G. Trambly de Laissardière, Phys. Rev. Lett. 71, 4166 (1993).

${ }^{30}$ M. Krajčí and J. Hafner, J. Phys.: Condens. Matter 12, 5831 (2000).

${ }^{31}$ K. Oshio and Y. Ishii, J. Alloys Compounds 341, 402 (2002).

${ }^{32}$ N. W. Ashcroft and N. D. Mermin, Solid State Physics (Holt, Rinehart and Winston, New York, 1976).

${ }^{33}$ S. E. Burkov, T. Timusk, and N. W. Ashcroft, J. Phys.: Condens. Matter 4, 9447 (1992).

${ }^{34}$ P. N. Butcher, Proc. Phys. Soc. (London) A 64, 756 (1951).

${ }^{35}$ A. I. Golovashkin, A. I. Kopeliovich, and G. P. Motulevich, Zh. Eksp. Teor. Fiz. 53, 2053 (1967) [Sov. Phys. JETP 26, 1161 (1968)]. 
${ }^{36}$ I. R. Fisher, Z. Islam, A. F. Panchula, K. O. Cheon, M. J. Kramer, P. C. Canfield, and A. I. Goldman, Phil. Mag. B 77, 1601 (1998).

${ }^{37}$ I. R. Fisher, K. O. Cheon, A. F. Panchula, P. C. Canfield, M. Chernikov, H. R. Ott, and K. Dennis, Phys. Rev. B 59, 308 (1999).
${ }^{38}$ A. Létoublon, I. R. Fisher, T. J. Sato, M. de Boissieu, M. Boudard, S. Agliozzo, L. Mancini, J. Gastaldi, P. C. Canfield, A. I. Goldman, and A.-P. Tsai, Mater. Sci. Eng. A 294-296, 127 (2000).

${ }^{39}$ H. Ehrenreich and H. R. Philipp, Phys. Rev. 128, 1622 (1962).

${ }^{40}$ H. Ehrenreich, H. R. Philipp, and B. Segall, Phys. Rev. 132, 1918 (1963). 